eISSN 2444-7986

DOI: https://doi.org/10.14201/orl20178.15304

\author{
Artículo original
}

\title{
RESULTADO FUNCIONAL Y SOCIAL DE LOS IMPLANTES AUDITIVOS OSTEOINTEGRADOS
}

\section{Functional and social results of osseointegrated hearing aids}

\author{
Inmaculada MORENO-ALARCÓN; Antonio BELINCHÓN-DIEGO \\ Complejo Hospitalario Universitario de Albacete. Servicio de Otorrinolaringología. España.
}

Correspondencia: inmillaorl@gmail.com

Fecha de recepción: 19 de noviembre de 2016

Fecha de aceptación: 12 de diciembre de 2016

Fecha de publicación: 17 de diciembre de 2016

Fecha de publicación del fascículo: 1 de junio de 2017

Conflicto de intereses: Los autores declaran no tener conflictos de intereses

Imágenes: Los autores declaran haber obtenido las imágenes con el permiso de los pacientes

Política de derechos y autoarchivo: se permite el autoarchivo de la versión post-print (SHERPA/RoMEO)

Licencia CC BY-NC-ND. Licencia Creative Commons Atribución-NoComercial-SinDerivar 4.0 Internacional

๔C Universidad de Salamanca. Su comercialización está sujeta al permiso del editor

RESUMEN

Introducción y objetivo: El implante osteointegrado constituye hoy en día una buena opción terapéutica en pacientes con hipoacusia de conducción o mixta. Los objetivos de este estudio son: valorar el beneficio audiológico de los pacientes con implante osteointegrado y cuantificar el cambio en su calidad de vida. Método: En el estudio se incluyó a 10 pacientes implantados entre marzo de 2013 y septiembre de 2014 en nuestro centro. El instrumento empleado para medir la calidad de vida fue el Glasgow Benefit Inventory (GBI) y un cuestionario con 3 preguntas: uso del implante, dolor postoperatorio y si recomendaría la intervención a otros pacientes. Para la valoración audiológica se realizó audiometría tonal y logoaudiometría en campo libre. Resultados: La puntuación media del Glasgow Benefit Inventory total fue +58 , y en las subescalas general, social y física $+75,+18$ y +29 , respectivamente. La mejoría con el implante respecto a la audiometría tonal en campo libre en las frecuencias de 500, 1000 y $2000 \mathrm{~Hz}$ resultó ser estadísticamente significativa, al igual que la diferencia entre la audiometría verbal antes y después del implante. Discusión: El avance en la técnica quirúrgica de este tipo de implantes, constituyendo actualmente una cirugía mínimamente invasiva, ha dado paso a la valoración de los aspectos funcional y social como medida de su eficacia. Conclusiones: El uso del implante osteointegrado se relaciona con una importante mejora a nivel audiológico, especialmente en pacientes con hipoacusia conductiva o mixta, junto a un gran cambio en calidad de vida de los pacientes implantados.

PALABRAS CLAVE BAHA; implante auditivo osteointegrado; hipoacusia de transmisión; calidad de vida; Glasgow Benefit Inventory

SUMMARY

Introduction and objective: Osseointegrated implants are nowadays a good therapeutic option for patients suffering from transmission or mixed hearing loss. The aims of this study are both to assess audiology benefits for patients with osseointegrated implants and quantify the change in their quality of life. Method: The study included 10 patients who were implanted in our hospital between March 2013 and September 2014. The instrument used to quantify their quality 
of life was the Glasgow Benefit Inventory $(\mathrm{GBI})$ and a questionnaire including three questions: use of implant, postoperative pain and whether they would recommend the operation to other patients. Audiology assessment was performed through tone audiometry and free field speech audiometric testing. Results: The average total benefit score with the Glasgow Benefit Inventory was +58 , and the general, social and physical scores were $+75,+18$ and +29 , respectively. The improvement with the implant regarding free-field tonal audiometry at the frequencies of 500,1000 and $2000 \mathrm{~Hz}$ was found to be statistically significant, as was the difference between verbal audiometry before and after implantation. Discussion: Improvements in surgical technique for osseointegrated implants, at present minimally invasive, foregrounds the assessment of functional and social aspects as a measure of their effectiveness. Conclusions: The use of the osseointegrated implant is related to an important improvement in the audiological level, especially in patients with conductive or mixed hearing loss, together with a great change in the quality of life of implanted patients.

KEYWORDS

BAHA; osseointegrated hearing aids; transmission hearing loss; quality of life; Glasgow Benefit Inventory

\section{INTRODUCCIÓN}

Los implantes de conducción por vía ósea se basan en la transformación de las ondas sonoras en vibraciones mecánicas que se transmiten, a través del hueso del paciente, hasta la cóclea, produciéndose así su estimulación.

La utilización de la vía ósea para tratar la hipoacusia ha sido utilizada desde hace años, inicialmente mediante instrumentos que el paciente sujetaba con los dientes, hasta prótesis que estimulan la zona mastoidea (diademas vibratorias o vibradores en las patillas de las gafas). El desarrollo tecnológico ha favorecido la aparición de los implantes osteointegrados actuales, que constan de un implante, generalmente de titanio, que mediante fresado se ancla al hueso temporal quedando posteriormente osteointegrado, y un procesador, que se encarga de captar el sonido y transformarlo en vibraciones. El procesador se une al implante mediante un pilar. La estimulación puede ser percutánea (el implante atraviesa la piel) o transcutánea (el implante está cubierto por la piel). Los implantes osteointegrados consiguen, respecto a los vibradores óseos externos clásicos (diademas y vibradores en las patillas de las gafas), una estimulación más directa del hueso y una mejor calidad y potencia del sonido.

El uso de este tipo de implantes está indicado principalmente en pacientes con hipoacusia conductiva o mixta con mala adaptación a las prótesis auditivas convencionales (escaso rendimiento, supuración frecuente).

La colocación del implante requiere un procedimiento quirúrgico que se realiza bajo anestesia local, poco invasivo y con una baja morbilidad (Figura 1). La Organización Mundial de la Salud define la salud como el completo estado de bienestar físico, mental y social. El éxito total de cualquier intervención quirúrgica no se puede obtener solamente del resultado técnico, también deben considerarse los cambios en la calidad de vida del paciente, lo que justifica el creciente número de estudios publicados al respecto. En el año 2001 el estudio publicado por Arunachalam et al., fue el primero en demostrar un beneficio significativo en la calidad de vida tras la cirugía del implante osteointegrado en los pacientes intervenidos, realizando la evaluación mediante el Glasgow Benefit Inventory [1].

Desde entonces hasta el momento actual han sido muchos los estudios publicados al respecto por diferentes grupos de trabajo, algunos de ellos utilizando el mismo cuestionario de calidad de vida que usó Arunachalam [2-8], y en otros casos se han utilizado otros métodos para evaluar el cambio en la calidad de vida de los pacientes [9-13]. Los resultados en todos ellos muestran una mejoría en la calidad de vida de los pacientes tras ser intervenidos de implante osteointegrado.

Los objetivos que pretendemos alcanzar con el presente estudio son:

1.- Valorar el beneficio audiológico del implante osteointegrado en nuestro centro.

2.- Valorar la calidad de vida de los pacientes con implante osteointegrado en nuestro centro.

\section{MATERIAL Y MÉTODO}

En el estudio se incluyeron 10 pacientes implantados de forma consecutiva entre marzo de 2013 y septiembre de 2014 en nuestro cen- 
tro, analizando los datos de forma retrospectiva. Un $60 \%$ eran varones y un $40 \%$ mujeres, con una media de edad de 64,3 años (rango de 45 a 80 ).

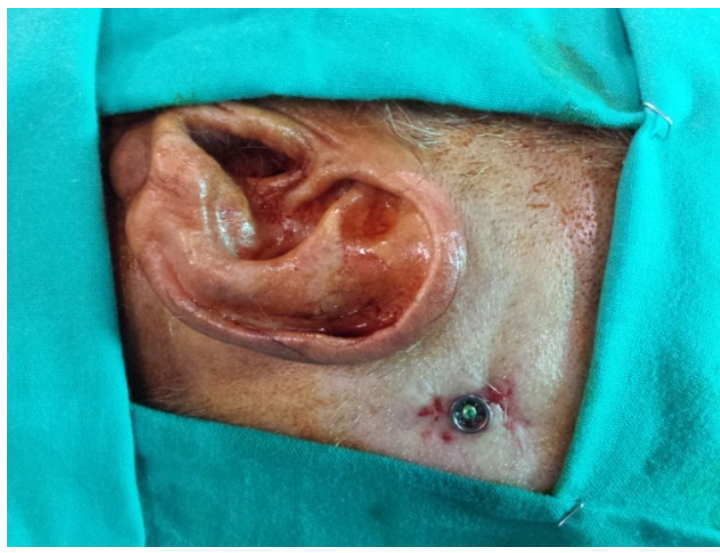

Figura 1. Imagen intraoperatoria del pilar del implante osteointegrado.

Respecto a la indicación atendiendo a los criterios otológicos, el $50 \%$ estaban diagnosticados de otitis media crónica (OMC), el 30\% tenían una OMC colesteatomatosa, $10 \%$ malformación del conducto auditivo externo (CAE) y $10 \%$ fístula de canal semicircular lateral (Tabla 1). La mitad se implantó en el lado derecho y la otra mitad en el lado izquierdo. Sólo uno de los pacientes había sido previamente portador de una prótesis de conducción por vía ósea (varillas auditivas).

Todos los pacientes fueron intervenidos bajo anestesia local. En todos ellos se colocó un implante osteointegrado percutáneo utilizando la técnica de la incisión lineal. Se realizó infiltración de anestesia local (lidocaína al $2 \%$ y adrenalina a 1/100.000) en la región temporal, posterosuperior al pabellón auricular, incisión lineal de aproximadamente $1 \mathrm{~cm}$ de longitud, centrada en la localización del pilar, y disección de los tejidos hasta periostio y hueso. Posteriormente se realizó el fresado y avellanado del hueso para la colocación del implante de titanio junto con el pilar. El procesador externo fue adaptado a los dos meses de la cirugía.

Para evaluar el beneficio auditivo con el implante, se realizó a todos los pacientes audiometría tonal en campo libre y logoaudiometría en campo libre. Ambas pruebas fueron realizadas en una cabina insonorizada, mediante altavoces.
Tabla 1. Causas otológicas de indicación del implante osteointegrado.

\begin{tabular}{|l|l|}
\hline & Número de pacientes \\
\hline Otitis media crónica & 5 \\
\hline $\begin{array}{l}\text { Otitis media crónica colestea- } \\
\text { tomatosa }\end{array}$ & 3 \\
\hline $\begin{array}{l}\text { Malformación conducto audi- } \\
\text { tivo externo }\end{array}$ & 1 \\
\hline $\begin{array}{l}\text { Fístula de canal semicircular } \\
\text { lateral }\end{array}$ & 1 \\
\hline & 10 \\
\hline
\end{tabular}

En el caso de la audiometría tonal, ésta ha sido realizada siguiendo el modo ascendente, iniciando la estimulación con intensidades débiles que se van aumentando en pasos de $5 \mathrm{~dB}$ hasta obtener la respuesta del sujeto. La primera frecuencia estudiada ha sido la de 1000 $\mathrm{Hz}$, seguida de las frecuencias más agudas, 2000,4000 y $8000 \mathrm{~Hz}$, y después las más graves, 500,250 y $125 \mathrm{~Hz}$.

La audiometría verbal o logoaudiometría ha sido realizada utilizando el método a viva voz, con listas de palabras estandarizadas, obteniendo la curva de inteligibilidad.

El instrumento empleado para medir la calidad de vida fue el Glasgow Benefit Inventory (GBI) [1] que es un cuestionario que mide los cambios en el estado de salud producidos por una intervención quirúrgica determinada. Consta de 18 preguntas que miden los cambios en la salud distinguiendo 3 campos: la salud general (12 preguntas), la social (3 preguntas) y la física (3 preguntas). Cada pregunta tiene una puntuación que oscila entre 1 y 5 , con una variación que va desde un gran deterioro en el estado de salud hasta una gran mejoría en el estado de salud del individuo. La puntuación total oscila entre 18 y 90 puntos, aunque el resultado numérico es transformado en una media (que oscila entre -100 y +100 ) que permite la comparación de los resultados entre subgrupos.

Así mismo, se preguntó acerca del uso del implante osteointegrado (horas por día), dolor con el implante (sí, no, a veces) y si el paciente lo recomendaría a otras personas (sí, no).

Se describieron los datos cuantitativos con medidas de tendencia central (mediana) y dispersión (intervalo intercuartílico: percentiles 25 y 75). Las comparaciones de los valores de las audiometrías, antes y después del implante, se realizaron con test no paramétricos (test de signos de Willcoxon). 
Los datos se analizaron con el paquete estadístico PASW 18.0 (SPSS Inc).

\section{RESULTADOS}

En el momento en que se realizó la evaluación de los pacientes dos de ellos habían fallecido, uno debido a un tumor pulmonar metastásico y otro, el más anciano de todos los pacientes implantados (80 años), por deterioro de su estado general tras una infección pulmonar.

Por todo ello, se analizaron un total de 8 pacientes, 4 hombres y 4 mujeres, con una edad mediana de 67,5 años (rango de 44 a 73 años). Para determinar la indicación según los criterios audiológicos se realizó a todos los pacientes, previo a la cirugía del implante, una audiometría tonal convencional cuyos resultados se muestran en la tabla 2.

Tabla 2. Resultados audiometría tonal convencional previa al implante osteointegrado.

\begin{tabular}{|clccc|}
\hline \multicolumn{1}{r}{ Vía } & Frecuencia & Mediana & P25 & P 75 \\
\hline \multirow{4}{*}{ Aérea } & $500 \mathrm{~Hz}$ & 78 & 70 & 85 \\
& $1000 \mathrm{~Hz}$ & 73 & 60 & 83 \\
& $2000 \mathrm{~Hz}$ & 70 & 63 & 80 \\
\hline \multirow{3}{*}{ Ósea } & $500 \mathrm{~Hz}$ & 35 & 30 & 55 \\
& $1000 \mathrm{~Hz}$ & 43 & 30 & 58 \\
& $2000 \mathrm{~Hz}$ & 48 & 40 & 68 \\
\hline
\end{tabular}

(P25: percentil 25; P75: percentil 75)

En cuanto a la valoración audiológica, comparando la audiometría tonal en campo libre antes y después de la colocación del implante osteointegrado (Tabla 3) obtuvimos una diferencia estadísticamente significativa, con un intervalo de confianza del $95 \%(p<0.05)$, en las frecuencias de 500, 1000 y $2000 \mathrm{~Hz}$.

Tabla 3. Audiometría tonal en campo libre, antes y después del implante osteointegrado.

\begin{tabular}{lccccccc} 
& \multicolumn{5}{c}{ Previa } & \multicolumn{5}{c}{ Con implante } & p \\
& Mediana & P25 & P 75 & Mediana & P25 & P 75 & \\
\hline $500 \mathrm{~Hz}$ & 53 & 30 & 73 & 25 & 15 & 28 & 0,012 \\
\hline $1000 \mathrm{~Hz}$ & 53 & 40 & 68 & 28 & 18 & 40 & 0,012 \\
\hline $2000 \mathrm{~Hz}$ & 63 & 58 & 70 & 50 & 38 & 55 & 0,011 \\
\hline
\end{tabular}

(P25: percentil 25; P75: percentil 75)

En el $50 \%$ de los pacientes la media de las frecuencias de 500, 1000 y $2000 \mathrm{~Hz}$ en la audiometría tonal en campo libre era $\geq 30 \mathrm{~dB}$.

Respecto a los resultados de la evaluación verbal mediante logoaudiometría, antes y después del implante, representados mediante diagrama de cajas (Figura 2), se observó una mejora de la inteligibilidad con el implante, estadísticamente significativa, con un intervalo de confianza del 95\% $(p<0.05)$.

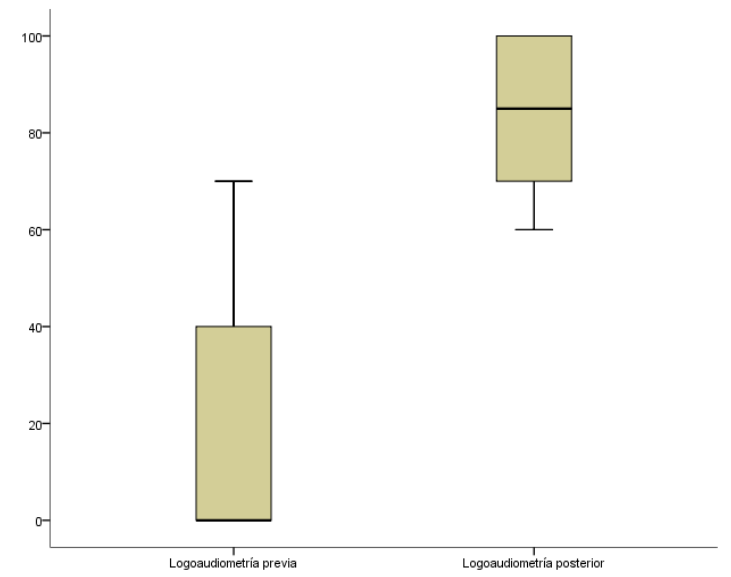

Figura 2. Diagrama de cajas de la logoaudiometría previa y posterior al implante $(p=0,011)$.

Contestaron el cuestionario GBI 8 pacientes (80\%), así como el cuestionario abierto.

La puntuación media del GBI total fue +58 , y en las subescalas general, social y física fue +75 , +18 y +29 , respectivamente. La tabla 4 muestra los datos cuantitativos con medida de tendencia central (mediana) y dispersión (intervalo intercuartílico: percentiles 25 y 75), respecto a los resultados del GBI.

Tabla 4. Descriptivos del Glasgow Benefit Inventory.

\begin{tabular}{|lccc|}
\hline & Mediana & P25 & P 75 \\
\hline Puntuación total & 59.72 & 50.00 & 66.66 \\
\hline Subescala General & 79.16 & 62.50 & 83.33 \\
\hline Subescala Social & 16.66 & .00 & 16.66 \\
\hline Subescala Física & 24.99 & 16.66 & 41.67 \\
\hline
\end{tabular}

(P25: percentil 25; P75: percentil 75)

La media de horas al día de utilización del implante osteointegrado por los pacientes fue de 12 horas, el $100 \%$ lo utilizaba los 7 días de la semana.

En lo referente al dolor en la zona del implante, sólo 1 paciente refirió tener molestias ocasionales.

El 100\% afirmó que recomendaría el implante a otros pacientes. 


\section{DISCUSIÓN}

El mayor beneficio audiológico con los implantes osteointegrados se obtiene en pacientes con hipoacusia de transmisión, siendo la mejora de la capacidad auditiva menor en caso de hipoacusias mixtas con un marcado componente neurosensorial $[3,8,11]$. Sin embargo, este último grupo podría representar un alto porcentaje de pacientes que se beneficiarían de este tipo de prótesis [7]. Al igual que en otros estudios, en el nuestro, el análisis por frecuencias de la mejora auditiva muestra una mayor mejoría de la audición en las frecuencias graves, siendo mayor la diferencia auditiva, con y sin implante, en 500 y $1000 \mathrm{~Hz}$ que, a partir de los $2000 \mathrm{~Hz}$, especialmente en los pacientes que presentaban en el estudio prequirúrgico pérdida auditiva neurosensorial en frecuencias agudas. A pesar de ello, la diferencia obtenida tras el implante en las tres frecuencias estudiadas $(500,1000$ y $2000 \mathrm{~Hz})$ es estadísticamente significativa en nuestra muestra.

Resulta importante señalar que, en algunos de nuestros pacientes, ha aparecido una sobreganancia entre la audiometría de conducción ósea convencional prequirúrgica, y la audiometría in situ con el implante osteointegrado puesto. Creemos que esto es debido a la atenuación que produce la piel sobre el vibrador en la prueba prequirúrgica, obteniendo, una vez colocado definitivamente el procesador, acoplado al implante percutáneo, una mayor ganancia auditiva que sorprende favorablemente a cirujano y paciente.

La repercusión de las actividades sanitarias sobre la calidad de vida de los pacientes adquiere una importancia cada vez mayor en el ejercicio de la medicina actual, incluso en muchas ocasiones por delante de los resultados puramente médicos, por lo que los cuestionarios que combinan la medida de la capacidad funcional, el bienestar psicosocial y el alivio sintomático, constituyen una herramienta importante para la cuantificación del nivel de salud de los pacientes. Actualmente, en este tipo de intervención, la técnica quirúrgica ha cedido importancia a los aspectos funcional y social, a partir de las intervenciones que no precisan reducción del tejido celular subcutáneo, lo que suponía el caballo de batalla de este tipo de implantes, que hoy día se realizan mediante una cirugía mínimamente invasiva, sencilla y con baja tasa de complicaciones [14]. Todo ello favorece que cobre mayor importancia el resultado psicosocial de la intervención que el resultado puramente quirúrgico (Figura 1).

La baja tasa de complicaciones derivada de la evolución de la técnica quirúrgica utilizada para la colocación de este tipo de implantes se traduce en nuestro estudio en que solamente un paciente presentó leves molestias en la zona del tornillo. Se considera que el dolor postoperatorio en estos casos podría estar en relación con el hecho de que, durante la intervención, parte del periostio quede incluido en el lecho del implante. Sin embargo, en nuestro paciente no encontramos este problema durante la cirugía, por lo que las molestias las atribuimos a causas desconocidas. El tratamiento en tal caso pasaría, primeramente, por la inyección de corticoide intralesional alrededor del tornillo y, si no es efectivo y las molestias o el dolor son muy intensos, en ocasiones sería necesaria la retirada del mismo.

La tasa de respuesta al cuestionario fue del $80 \%$, comparable a otros estudios similares realizados previamente [1, 3, 5] (89\% Sánchez-Camón, 73\% McLarnon, 85\% Arunachalam).

Comparando los valores del GBI con los de otros procedimientos quirúrgicos otológicos para mejora de la audición, destaca que el implante osteointegrado produce una mayor mejoría en la calidad de vida que la cirugía del oído medio [15], y sólo algo menos que el implante coclear [16]. Estos datos, junto con las consideraciones económica y de complicaciones técnicas del procedimiento, pondrían a los implantes osteointegrados como una muy buena opción de tratamiento para pacientes con hipoacusia.

La mayor ganancia de calidad de vida se produce en el aspecto general o global de la persona, al igual que ocurre con los estudios realizados en calidad de vida de los implantes cocleares [16]. Destaca que el implante osteointegrado influye poco en el aspecto somático de la calidad de vida, ya que no es éste el objetivo de este tipo de procedimiento, que lo que busca es una mejora de la capacidad auditiva del paciente.

Sin embargo, estudios realizados en otros procedimientos otorrinolaringológicos, como la amigdalectomía, muestran resultados diferentes de calidad de vida en cuanto al beneficio por subescalas del GBI, obteniendo los mejores resultados en el aspecto físico, en contraposición del beneficio general y social del procedimiento [17, 18], debido a que el objetivo que se pretende alcanzar en este caso sí que 
es una mejora en la sintomatología física del individuo.

Los estudios realizados en calidad de vida en pacientes sometidos a cirugía de schwannoma vestibular muestran datos totalmente opuestos, demostrando en la mayoría de los pacientes un deterioro en su calidad de vida [19], esto puede ser debido a que los síntomas iniciales de esta patología suelen ser leves y, sin embargo, las secuelas postquirúrgicas pueden ser muy invalidantes.

Los datos obtenidos en nuestro estudio sobre la calidad de vida son comparables a los de otros estudios previos en cuanto a beneficio general del implante osteointegrado, con la limitación del escaso número de pacientes de nuestra serie. Medido por subescalas, nuestros resultados incluso mejoran los estudios anteriores, posiblemente, respecto a estudios más antiguos, por la evolución de la técnica quirúrgica empleada, que, dada su sencillez y el menor número de curas necesarias en el postoperatorio, comparada con la técnica del colgajo y reducción de tejido subcutáneo, hace que los pacientes sean más susceptibles a la hora de recomendar este procedimiento a potenciales usuarios.

Resulta muy significativo el hecho de que todos los pacientes recomendarían el uso del implante osteointegrado a otra persona, al igual que sucede en otras series, siendo un dato más a favor de los buenos resultados obtenidos con este tipo de implantes respecto al cambio en la calidad de vida tras la cirugía.

\section{CONCLUSIONES}

Los resultados audiológicos no son suficientes para valorar de forma adecuada el beneficio que los pacientes obtienen del implante osteointegrado, imponiéndose la necesidad de utilizar cuestionarios de calidad de vida para una correcta valoración de estos pacientes, lo que tiene implicaciones para los criterios de selección y el asesoramiento preoperatorio de los pacientes candidatos a este tipo de cirugía. El uso del implante osteointegrado se asocia a una importante mejoría en la calidad de vida de los pacientes. La ganancia audiológica es mayor en frecuencias graves. Las mejoras en la técnica quirúrgica del implante osteointegrado percutáneo junto con la ganancia obtenida en calidad de vida hacen, que este tipo ayuda auditiva, sea muy recomendable desde los pacientes implantados hacia los pacientes que potencialmente se van a implantar.

\section{BIBLIOGRAFÍA}

1. Arunachalam PS, Kilby D, Meikle D, Davison T, Johnson IJM. Bone-Anchored hearing aid quality of life assessed by Glasgow Benefit Inventory. Laryngoscope. 2001;111:1260-1263.

2. Dutt $S N$, McDermott $A L$, Jelbert $A$, Reid AP, Proops DW. The Glasgow benefit inventory in the evaluation of patient satisfaction with the bone-anchored hearing aid: quality of life issues. J Laryngol Otol. 2002;116(28):7-14.

3. McLarnon CM, Davison T, Johnson IJM. Bone-Anchored hearing aid: comparison of benefit by patient subgroups. Laryngoscope. 2004;114:942-4.

4. Gillett D, Fairley JW, Chandrashaker TS, Bean A, González J. Bone-anchored hearing aids: results of the first eight years of a programme in a district general hospital, assessed by the Glasgow benefit inventory. J Laryngol Otol. 2006;120:537-542.

5. Sánchez-Camón I, Lassaletta L, Castro A, Gavilán J. Calidad de vida de los pacientes con BAHA. Acta Otorrinolaringol Esp. 2007;58(7):316-20.

6. Ho EC, Monksfield P, Egan E, Reid A, Proops D. Bilateral Bone-Anchored Hearing Aid: Impact on Quality of Life Measured with the Glasgow Benefit Inventory. Otol Neurotol. 2009; 30: 891-6.

7. Wazen JJ, Van Ess MJ, Alameda J, Ortega C, Modisett M, Pinsky K. Otolaryngol Head Neck Surg. 2010;142:554-9.

8. Lekue A, Lassaletta L, Sánchez-Camón I, Pérez-Mora R, Gavilán J. Calidad de vida de pacientes implantados con el dispositivo BAHA según su indicación. Acta Otorrinolaringol Esp. 2013;64(1):17-21.

9. Myrthe KS Hol, Marian A Spath, Paul FM Krabbe, Catharina TM van der Pouw, Ad FM Snk, Cor WRJ Cremers et al. The Bone-Anchored Hearing Aid. Quality of life assessment. Arch Otolaryngol Head Neck Surg 2004;130:394-9.

10. Badran K, Bunstone D, Arya AK, Suryanarayanan R, Mackinnon N. Patient Satisfaction with the Bone-Anchored Hearing Aid: A 14-Year Experience. Otol Neurotol. 2006;27:659-66. 
11. Saroul N, Gilain L, Montalban A, Giraudet $F$, Avan $P$, Mom $T$. Patient satisfaction and functional results with the bone-anchored hearing aid (BAHA). Eur Ann Otorhinolaryngol Head Neck Dis. 2011;128:107-13.

12. Gardell ISK, Andresen K, Faber CE, Wanscher $\mathrm{JH}$. Bone-anchored hearing aids are effective and associated with a high degree of satisfaction. Dan Med J. 2015;62(7):A5108.

13. Nelissen RC, den Besten CA, Faber HT, Dun CAJ, Mylanus EAM, Hol MKS. Loading of osseointegrated implants for bone conduction hearing at 3 weeks: 3 year stability, survival and tolerability. Eur Arch Otorhinolaryngol. 2016;273:1731-7.

14. Ricci G, Della Volpe A, Faralli F, Longari $F$, Gullà M, Mansi N et al. Results and complications of the Baha system (bone-anchored hearing aid). 2010;267:1539-45.

15. Subramaniam K, Eikelboom RH, Marino $\mathrm{R}$, Atlas MD, Rajan GP. Patient's quality of life and hearing outcomes after stapes surgery. Clin Otolaryngol. 2006;31:2739.
16. Castro A, Lassaletta L, Bastarrica M, Alfonso $C$, Prim MP, de Sarriá MJ et al. Calidad de vida en pacientes con implante coclear. Acta Otorrinolaringol Esp. 2005;55:192-7.

17. Schwentner I, Höfer S, Schmutzhard J, Deibl M, Sprinzl GM. Impact of tonsillectomy on quality of life in adults with chronic tonsillitis. Swiss Med Wkly. 2007; 137:454-61.

18. Senska G, Atay $H$, Pütter $C$, Dost $P$. Long-Term Results from Tonsillectomy in Adults. Dtsch Arztebl Int. 2015;112:849-55.

19. Alfonso C, Lassaletta L, Sarriá J, Gavilán J. Calidad de vida tras la cirugía del schwannoma vestibular. Acta Otorrinolaringol Esp. 2007;58(2):61-5.

\section{ENLACES RELACIONADOS}

Sánchez-Cuadrado I, Lassaletta L, PérezMora R, Muñoz E, Gavilán J. Reliability and validity of the Spanish Glasgow Benefit Inventory after cochlear implant surgery in adults. European Archives of Oto-Rhino-Laryngology. Springer Nature; 2013 Dec 13;272(2):333-6. DOI: dx.doi.org/10.1007/s00405-013-2844-y. 